Revue internationale P.M.E.

Économie et gestion de la petite et moyenne entreprise

Revue

internationale

PME

\title{
Devancer la concurrence par les systèmes d'information
}

\section{François Bergeron et Chantal Buteau}

Volume 1, numéro 3-4, 1988

URI : https://id.erudit.org/iderudit/1007886ar

DOI : https://doi.org/10.7202/1007886ar

Aller au sommaire du numéro

Éditeur(s)

Presses de l’Université du Québec

ISSN

0776-5436 (imprimé)

1918-9699 (numérique)

Découvrir la revue

Citer cet article

Bergeron, F. \& Buteau, C. (1988). Devancer la concurrence par les systèmes d'information. Revue internationale P.M.E., 1(3-4), 295-307.

https://doi.org/10.7202/1007886ar

\section{Résumé de l'article}

Les systèmes d'information à avantages concurrentiels (SIAC) font partie des systèmes d'information où la technologie informatique est génératrice de profits. Le but de cet article est de présenter un modèle permettant l'identification de SIAC au sein des moyennes entreprises, Ce modèle met l'emphase sur le développement d'une vision stratégique à l'intérieur de la moyenne entreprise en vue de mieux exploiter les opportunités créées par la technologie de l'information. Il est basé sur une grille d'analyse mettant en perspective les cibles stratégiques et les stratégies concurrentielles que peut développer une entreprise en regard de ses principales activités. 


\title{
Devancer la concurrence par les systèmes d'information
}

\author{
François BERGERON* \\ Chantal BUTEAU** \\ Université Laval
}

\begin{abstract}
RÉSUME
Les systèmes d'information à avantages concurrentiels (SIAC) font partie des systèmes d'information où la technologie informatique est génératrice de profits. Le but de cet article est de présenter un modèle permettant l'identification de SIAC au sein des moyennes entreprises. Ce modèle met l'emphase sur le développement d'une vision stratégique à l'intérieur de la moyenne entreprise en vue de mieux exploiter les opportunités créées par la technologie de l'information. II est basé sur une grille d'analyse mettant en perspective les cibles stratégiques et les stratégies concurrentielles que peut développer une entreprise en regard de ses principales activités.
\end{abstract}

\begin{abstract}
The purpose of this study is to test a methodology to plan for the implementation of information systems for competitive advantage in medium size organizations. The methodology uses a grid that puts in perspective the strategic thrusts, the strategic targets and the main organizational activities. Results indicate that 1 - it is possible to identify opportunities of information systems for competitive advantage in organizations, and 2-most of these opportunities constitute worthy investments. Each opportunity was assessed in terms of costs, revenues, decision of implementation and time of implementation.
\end{abstract}

- François Bergeron est professeur agrégé à la Faculté des sciences de l'administration de l'Université Laval. Il est directeur du réseau de Recherche en Informatisation des Organisations (RIO). Il détient un $\mathrm{Ph}$.D en systèmes d'information de l'Université de Califormie, Los Angeles (U.C.L.A.). M. Bergeron a publié plusieurs articles dans des revues telles MIS Quarterly, Revue Canadienne des Sciences de l'Administration et Gestion. Il est aussi l'auteur de volumes en systèmes d'information dont le demier «L'informatisation dans les PME: douze cas types» a été publié conjointement avec $L$. Raymond et S. Rivard.

* Chantal Buteau détient un MBA del'Université Laval et a travaillé comme professionnelle de recherche à la Faculté des sciences de l'administration. Elle travaille maintenant comme conseillère en systèmes d'information avec le Groupe CGI de Québec.

(Adresse: Faculté des sciences de l'administration, Université Laval, Québec, G1K 7P4). Premier manuscrit reçu en juin 1988. 


\section{RESUMEN}

Los sistemas de información con ventajas competitivas hacen parte de los sistemas de informaciōn donde la tecnologia informatizada es generadora de ganancias. El objetivo de este articulo es de presentar un modelo que permita la identificacion de «SIAC w al seno de las empresas medianas. Este modelo pone enfasis en el desarollo de una vision estrategica en el interior de la mediana empresa en vista de una mejor explotaciōn de las oportunidades creadas por la tecnologia de la computaciōn. Este se basa en un formulario de analisis que mete en perspectiva los objectivos estrategicos y los estrategias competitivas que puede dessarollar una empresa a partir de sus principales activades. Los resultados preliminares de la aplicaciōn del modelo cerca de cinco empresas manufactureras revelan su eficacia.

\section{Introduction}

On sait qu'antérieurement, les moyennes entreprises(M.E.)n'ont pu adopter un taux d'informatisation aussi rapide que les grandes entreprises en raison des coûts élevés de l'équipement. Cependant, la diminution des coûts engendrée par la venue de la micro-informatique a contribué à faciliter la pénétration de l'informatique dans ces entreprises dans les dernières années. En plus de la baisse des coûts de la microinformatique, le marché profite désormais de l'apparition de systèmes d'information à avantages concurrentiels (SIAC). Les SIAC sont des systèmes qui permettent à l'entreprise d'obtenir une rente financière sous la forme d'une valeur ajoutée aux produits ou services mis en marché. Ces systèmes d'information affectent la position concurrentielle de l'organisation de différentes façons : $1^{\circ}$ en changeant la structure de l'industrie et ses règles du jeu; $2^{\circ}$ en créant un avantage concurrentiel par l'adoption de nouveaux moyens de dépasser les concurrents et $3^{\circ}$ en créant des opportunités pour de nouvelles affaires à l'intérieur même des opérations courantes de l'organisation (Porter et Millar, 1985). Nous proposons une méthode permettant l'identification des systèmes d'information à avantages concurrentiels au sein des moyennes entreprises. Cet article est divisé en cinq parties principales. Suite à cette introduction, la partie II discute du concept même du SIAC, la partie III porte sur la méthodologie suggérée de planification du SIAC et la partie IV présente quelques résultats de l'application de cette méthode auprès d'un échantillon de cinq moyennes entreprises. Le tout se termine par une mise en perspective

\section{Le concept de systèmes à avantages concurrentiels (SIAC)}

Les systèmes d'information à avantages concurrentiels sont des systèmes informatiques qui permettent d'aider ou de formuler la stratégie concurrentielle de l'organisation et ce, en vue d'obtenir (ou de maintenir) un avantage concurrentiel (Wiseman, 1985). Ilsconcourentégalement aux objectifs fondamentaux de l'entreprise 
et peuvent avoir un impact significatif sur son développement (EDP Analyzer, 1984). Plus précisément, ces systèmes peuvent être conçus pour aider une organisation, par exemple, à fabriquer au coût le moins élevé, à se distinguer des concurrents ou à se spécialiser sur un segment de marché particulier (créneau) (Porter, 1980; Porter et Millar, 1985). Ces trois stratégies peuvent être accomplies en utilisant la technologie de l'information pour ériger des barrières à l'entrée, augmenter le pouvoir de négociation avec les fournisseurs, créer de nouvelles dépendances avec les clients, offrir de nouveaux produits ou des produits substituts de même que pour changer la base de la concurrence ou la nature de celle-ci (Porter, 1979; McFarlan, 1984; Porter, 1985b). Toute stratégie concurrentielle doit tendre à réal iser un avantage concurrentiel soutenable. Un tel avantage est obtenu lorsque la différenciation est perceptible aux yeux des consommateurs, lorsque la firme a une capacité de maintenir cette différenciation par rapport à ses concurrents et lorsque cette différenciation persiste dans le temps (Coyne, 1986). La présence d'un avantage concurrentiel doit normalement se traduire en un apport financier supplémentaire à long terme pour l'organisation.

Ce concept a été bien exploité, par exemple, par les Cuisines MKS Inc., Publico Inc. et l'Encan Électronique. Les Cuisines MKS Inc. sont une compagnie québécoise de taille moyenne oeuvrant dans la fabrication d'armoires de cuisine. Pour faciliter le travail de ses détaillants dans la réalisation de devis pouvant exiger jusqu'à quatre heures de travail, Cuisines MKS a mis au point un système de type CAO (Conception Assistée par Ordinateur). Ce logiciel permet de créer en moins de dix minutes un devis tri-dimensionnel de la cuisine du client montrant les différents agencements d'armoires et de comptoirs possibles. Le logiciel produit une facture détaillée des coûts associés à l'agencement choisi. Ce système a permis à MKS de prendre une forte expansion sur son territoire, alors que les concurrents ne possédant pas un tel système en étaient encore aux devis faits à la main.

Un autre exemple est la compagnie Publico. Celle-ci, fondée en 1978, s'est spécialisée dans la publication d'appels d'offres informatisés (Raymond, Rivard et Bergeron, 1988). Ce système baptisé SIAO (Service Informatisé d'Appels d'Offres) a été établi après uneétude de faisabilité très approfondie. Il permet aux entrepreneurs de prendre connaissance des appels d'offres des différents employeurs (ministères, municipalités, etc.) parle biais d'un terminal informatique plutôt que parl' intermédiaire de revues spécialisées. LeSIAO a connu une grande popularitéauprès des entrepreneurs qui ont maintenant accès à plus d'information sur un plus grand nombre d'offres et ce, sans délai. Les abonnés au système disposent donc de plus de temps que leurs concurrents pour préparer leurs soumissions et c'est ce qui explique le succès du système SIAO

Un dernier exemple est l'Encan Électronique. Cette nouvelle entreprise québécoise est spécialisée dans la vente de porcs. L'Encan Électronique permet aux producteurs de porcs d'inscrire leurs porcs à un système central qui les met en vente à taux décroissant. Les communications entre l'Encan et l'acheteur se font de façon électronique. Les porcs sont vendus au plus offrant et l'Encan choisit alors parmi ses 
membres lequel d'entre eux vendra ses porcs de façon à minimiser les coûts de transport entre le producteur et l'acheteur. Comparativement à un encan traditionnel où le bétail doit être transporté sur les lieux et où les prix peuvent varier considérablement, l'Encan Électronique permet de stabiliser les prix, de toucher des clientèles nouvelles, par exemple l'Ontario et les États-Unis, et de minimiser ainsi les frais de transport.

Il existe plusieurs méthodes pour identifier des SIAC dans les organisations. Les plus connues sont l'analyse des forces compétitives (Porter, 1979; McFarlan, 1984), des stratégies compétitives (Porter, 1980; Porter et Millar, 1985), de la chaîne de valeur (Porter, 1985a; Porter, 1985b; Porter et Millar, 1985), la théorie des options stratégiques (Wiseman, 1985; Wiseman et MacMillan, 1984; Rackoff et al., 1985), le processus d'acquisition d'un produit par le consommateur (Ives et Learmonth, 1984; Learmonth et Ives, 1987), l'impact de la technologie de l'information (Parsons, 1983; Munro et Huff, 1985) et la matrice des opportunités stratégiques (Benjamin et al., 1984). Cependant, ces méthodes n'offrent que peu d'explications quant à la façon de démarrer le processus d'identification des SIAC dans les entreprises, donnent peu de conseils en regard du processus d'identification des applications et sont généralement très vagues relativement aux personnes devant être impliquées et aux activités à accomplir (Bergeron, Buteau et Raymond, 1989). De façon générale, elles se limitent à un cadre de travail plutôt théorique que pratique.

Les méthodes actuelles pour identifier un SIAC ont été conçues pour la grande entreprise. Or la moyenne entreprise possède certaines spécificités qui la différencient de la grande et il faudrait que ces méthodes soient adaptées à elle. Les M.E. possèdent des avantages particuliers qu'elles peuvent exploiter, dont ceux reliés à leur taille et à leur flexibilité, à la rapidité d'implantation des décisions, à leur capacité d'adaptation et de réorientation à court terme et à la proximité et fidélité de leurs marchés (Dupont, 1986). D'un autre côté, les M.E. manquent généralement de ressources financières et humaines (Raymond et al., 1988) et d'information fiable pour bien analyser la firme et son environnement (marché, concurrents, position stratégique). De plus, le nombre limité des produits qu'elles offrent ne sont souvent destinés qu'à un marché spécifique contribuant encoreà augmenter leur vulnérabilité. Enfin, compte tenu de leur taille, elles ont peu de facilité à contrôler les événements reliés à l'incertitude de leur environnement.

Quant à l'usage de l'informatique dans les M.E., il existe un fort potentiel d'utilisation de cette technologie tant aux niveaux opérationnel et comptable que décisionnel et stratégique (Raymond, 1984). Cependant, force nous est de constater que la majorité des applications informatiques sont développées pour des raisons d'ordre tactique plutôt que stratégique (Raymond, Rivard et Bergeron, 1988). Les systèmes d'information supportant des stratégies à long terme ou permettant de concevoir de nouvelles stratégies sont en effet moins courants dans la M.E. que dans la grande entreprise. Cela est compréhensible car la M.E. a moins tendance que la grande entreprise à planifier son développement à long terme. 
L'absence de système d'information à caractère stratégique est peut-être due à la difficulté de planifier les activités d'une M.E. De façon générale, la planification doit être adaptée aux particularités de la moyenne entreprise. En ce sens, il est préférable d'utiliser le minimum de ressources, de rester cohérent avec le style de gestion intuitif du dirigeant et de l'appuyer de l'examen des objectifs, des stratégies et de l'orientationà adopter pour son entreprise. D'autre part, la planification dans les M.E. doit faire appel à des services externes qui fourniront l'expertise nécessaire pour aider les gestionnaires à mettre l'accent sur les aspects critiques de l'entreprise. La planification de SIAC pour une M.E. exige que l'on revoit la planification organisationnelle de façon à ce que les objectifs poursuivis par les systèmes s'agencent adéquatement avec les objectifs organisationnels et vice-versa.

Les méthodes actuelles de planification des SIAC dans les entreprises ne tiennent pas compte des particularités des M.E. et, de façon générale, ne donnent que peu de conseils en regard du processus d'identification des applications et des personnes à impliquer. La prochaine partie présente une méthode de planification des SIAC dans les M.E. qui tient compte de leur spécificité.

\section{$3 \quad$ Méthodologie de planification des SIAC}

Pour identifier des opportunités de SIAC dans une M.E., nous proposons une approche constituée de cinq étapes, soit: $1^{\circ}$ la sensibilisation préalable; $2^{\circ} 1^{\prime}$ initiation aux SIAC; $3^{\circ} 1^{\prime}$ analyse de l'organisation; $4^{\circ} 1^{\prime}$ application de la grille et $5^{\circ} 1^{\prime}$ 'évaluation des autres possibilités.

Fig. 1. Étapes du processus de planification de SIAC

1. Sensibilisation préalable

2.Initiation aux SIAC

3. Analyse de l'organisation

4. Application de la grille d'identification des SIAC

5. Évaluation des autres possibilités 


\subsection{Sensibilisation préalable}

Tout d'abord, il est important que la direction de la M.E. perçoive un besoin de changement car son attitude vis-à-vis l'innovation joue un rôle majeur (Julien, Carrière et Hébert, 1988). Pour sensibiliser les gestionnaires aux bénéfices que peut apporter la technologie informatique, un comité des hauts gestionnaires doit être formé. Il regroupe ordinairement le dirigeant principal, les personnes responsables de l'informatique et de la planification stratégique ainsi que les individus oeuvrant dans les secteurs clés de l'entreprise, notamment dans les domaines du marketing et de la production. Une étroite collaboration de la part de ces individus permettra de découvrir des applications informatiques liées à leurs préoccupations. La participation de ces gestionnaires facilitera aussi l'implantation des SIAC identifiés car ils auront été impliqués dans le processus. La présence d'un consultant est aussi suggérée. Ce consultant servira de catalyseur des efforts des gestionnaires dans l'identification de SIAC pour l'entreprise.

\subsection{Initiation aux systèmes d'information à avantages concurrentiels}

L'initiation aux SIAC permetaux membres de l'organisation de se familiariser avec ce nouveau concept. Ceci se fait par une explication claire mais sommaire de ces systèmes, des fonctions qu'ils effectuent de même que des avantages concurrentiels retirés de leur utilisation. Cette étape favorise la compréhension du concept de SIAC, des différences avec les autres types de systèmes d'information, et du rôle que peuvent jouer les SIAC pour supporter ou formuler la stratégie de l'entreprise. Cette introduction permet aussi de constater la diversité des applications concurrentielles rendues possibles par la technologie de l'information. La compréhension du concept de SIAC est facilitée par l'utilisation d'exemples de systèmes d'information implantés dans d'autres entreprises. Cette étape est très importante car elle permet de sensibiliser les gestionnaires à l'importance que pourrait avoir l'informatique au sein de leur entreprise.

\subsection{Analyse de l'organisation}

Il est nécessaire d'obtenir une bonne compréhension de l'organisation, de ses activités et de son environnement afin d'identifier des systèmes adaptés à la stratégie que poursuit l'entreprise. Cette étape relève donc de la planification stratégique. Les gestionnaires aidés par le consultant doivent d'abord établir un portrait de l'organisation. Cela s'effectue par une revue des objectifs poursuivis par la firme, de sa stratégie de croissance, des produits fabriqués et par une identification des clients, fournisseurs et concurrents. A ce stade, il est primordial d'identifier les forces et faiblesses de l'entreprise de même que les menaces auxquelles elle fait face et les opportunités dont elle pourrait tirer avantage. L'étude de l'organisation est complétée par l'analyse des facteurs de l'environnement (contrôlables ou non) qui affectent ses activités. 


\subsection{Application de la grille d'identification des SIAC}

L'identification des SIAC se fait par l'entremise de la grille stratégique (figure 2). Cette grille est composée de trois axes : a) les cibles stratégiques, b) les stratégies concurrentielles et $\mathrm{c}$ ) les activités organisationnelles.

\section{a) cibles stratégiques}

L'identification de SIAC nécessite une connaissance préalable de trois principaux groupes faisant partie de l'environnement de l'organisation et qui constituent des cibles potentielles vers lesquelles peuvent être dirigées les applications informatiques concurrentielles. Les relations qu'entretient la firme avec ces groupes peuvent être soumises à une forte concurrence. La technologie de l'information peut alors être utilisée de plusieurs façons pour améliorer les perceptions des clients et des fournisseurs de même que la position concurrentielle de l'entreprises face à ses concurrents. Les fournisseurs incluent toutes les entreprises qui fournissent les matières et les services nécessaires à la création, à la transformation et/ou à la vente du produit ou service au consommateur. Les concurrents comprennent toutes les organisations qui vendent ou qui peuvent potentiellement vendre des produits jugés comme étant similaires ou substituts aux produits de la firme. Finalement, les organisations qui achètent le produit ou le service pour leur propre utilisation (utilisateur final) ou tout simplement pour le revendre (grossistes, distributeurs, détaillants) constituent les clients de l'entreprise.

\section{b) stratégies concurrentielles}

Les stratégies concurrentielles sont des orientations offensives ou défensives que peut adopter une entreprise à la recherche d'un avantage concurrentiel. Ce sont elles qui permettent de relier la stratégie que poursuit la firme à la technologie des systèmes d'information. La différenciation permet à la firme de distinguer ses produits ou services face à ses fournisseurs, sesclients ou ses concurrents. L'adoption de la stratégie de coût lui permettra de réduire ou d'éviter des coûts que la compagnie devrait normalement assumer. Elle pourrait aussi bénéficier d'un traitement préférentiel en tentant de réduire les coûts que doivent encourir ses fournisseurs ou ses clients. Finalement, la stratégie de croissance devrait permettre à la compagnie d'identifier de nouveaux produits, de nouveaux marchés, de satisfaire des besoins additionnels des consommateurs, d'adopter de nouvelles technologies, d'identifier ou de se concentrer dans un «créneau»particulier.

\section{c) activités organisationnelles}

Les activités organisationnelles représentent des éléments de la chaîne de valeur. Ils sont regroupés par cible stratégique pour faciliter l'identification des SIAC selon chaque cible. Elles ne sont pas exclusives à chaque cible stratégique mais sont regroupées selon leur appartenance la plus commune. 
Fig. 2. Grille stratégique pour l'identification de SIAC

\begin{tabular}{|c|c|c|c|}
\hline $\begin{array}{l}\text { Stratégies } \\
\text { concurrentielles }\end{array}$ stratégiques & Fournisseurs & Concurrents & Clients \\
\hline Différenciation & $\begin{array}{l}\text { 1. Système de } \\
\text { communication } \\
\text { électronique avec } \\
\text { les fournisseurs } \\
\text { (alimentation) }\end{array}$ & $\begin{array}{l}\text { 2- Atelier } \\
\text { flexible de } \\
\text { fabrication de } \\
\text { meubles }\end{array}$ & $\begin{array}{l}\text { 3- Conception } \\
\text { de chaussures } \\
\text { assistée par } \\
\text { ordinateur } \\
\text { selon les goûts } \\
\text { du grossiste }\end{array}$ \\
\hline Coût & $\begin{array}{l}\text { 4- Système } \\
\text { informatisé } \\
\text { sur les } \\
\text { transporteurs }\end{array}$ & $\begin{array}{l}\text { 5- Vendeurs } \\
\text { munis de } \\
\text { micro- } \\
\text { ordinateurs } \\
\text { portatifs }\end{array}$ & $\begin{array}{l}\text { 6- Système infor- } \\
\text { matisé permet- } \\
\text { tant le suivi } \\
\text { des commandes } \\
\text { par les détail- } \\
\text { lants }\end{array}$ \\
\hline $\begin{array}{l}\text { Croissance } \\
\text { (créneau) }\end{array}$ & (1) & $\begin{array}{l}\text { 7- Base de } \\
\text { données } \\
\text { marketing }\end{array}$ & (2) \\
\hline $\begin{array}{l}\text { Activités } \\
\text { organisationnelles }\end{array}$ & $\begin{array}{ll}\text { - } & \text { Manipulation M.P. } \\
\text { - } & \text { Contrôle des } \\
\text { stocks } \\
\text { - } \\
\text { - } & \text { Transprovisionnement } \\
\text { - } & \text { Service aux } \\
& \text { fournisseurs }\end{array}$ & $\begin{array}{ll}\text { - } & \text { Production } \\
\text { - Entretien } \\
\text { d'équipement } \\
\text { - } & \text { Manutention } \\
\text { et entreposage } \\
\text { - R \& D } \\
\text { - CTB, Finance } \\
\text { - Gestion, ADM } \\
\text { - Planification }\end{array}$ & $\begin{array}{ll}\text { - } & \text { Entrée des } \\
\text { commandes } \\
\text { - } & \text { Autorisation } \\
\text { du crédit } \\
\text { - } \quad \text { Force de vente } \\
\text { - } \quad \text { Circuit de } \\
\text { distribution } \\
\text { - } \quad \text { Fixation des } \\
\text { prix } \\
\text { - Etude de marché } \\
\text { - Etude de marché } \\
\text { - } \quad \text { Livraison } \\
\text { - } \quad \text { Service aux } \\
\text { clients } \\
\text { - Facturation }\end{array}$ \\
\hline
\end{tabular}

(1) Au centre de la grille se trouvent quelques exemples de SIAC identifiés dans quelques moyennes entreprises.

(2) Aucun système de ce type n'a été identifié. 
Pour appliquer la grille d'identification des SIAC, le consultant doitd'abord expliquer les divers concepts qui la sous-tendent (cibles stratégiques, stratégies concurrentielles et activités de l'organisation). Par la suite, le groupe de gestionnaires procède à l'identification d'applications informatiques pouvant procurer un avantage concurrentiel à la firme. Cette activité s'effectue à l'aide d'une série de questions soulevées à l'intérieur des (9) cases de la grille. Ainsi, pour chacune des cibles et des options stratégiques, il faut s'interroger sur la façon dont il serait possible d'utiliser la technologie de l'information en vue d'obtenir un avantage concurrentiel tout en se préoccupant du fonctionnement de l'entreprise dans ses différentes activités.

\section{5 Évaluation des autres possibilités}

Après l'étape d'identification des systèmes, une courte étude de faisabilité est réalisée. Elle porte sur l'évaluation des coûts de développement et d'implantation, sur les bénéfices et les risques associés à l'implantation ainsi que sur la décision ou non d'implanter de tels systèmes.

Chacune des étapes de la méthode, à savoir l'initiation au concept des SIAC, l'analyse de l'organisation, l'application de la grille et l'évaluation des autres possibilités, doit faire l'objet d'une session intensive. Cependant, il se peut que l'étape d'analyse de l'organisation nécessite certains délais relatifs à la collecte d'informations pertinentes. Aussi, certaines sessions devraient idéalement être espacées dans le temps, permettant alors à chacun des membres de réfléchir davantage au concept, de développer les habilités nécessaires à une vision stratégique et de diffuser les informations pertinentes vers les niveaux hiérarchiquement inférieurs.

\section{$4 \quad$ Les résultats}

Cette section présente quelques résultats obtenus de l'application de la méthode auprès de cinq entreprises. Ces résultats sont présentés de façon sommaire et visent surtout à illustrer le genre de produits auxquels peuvent s'attendre les entreprises qui désirent appliquer la méthode. Ils ne constituent pas une preuve scientifique de son efficacité mais plutôt une justification par des exemples. L'étude fut menée auprès de cinq entreprises québécoises de taille moyenne, comptant entre 200 et 350 employés et oeuvrant dans des secteurs manufacturiers différents, soit le cuir, le meuble, le papier, l'alimentation et les produits chimiques. 


\subsection{Nombre de systèmes identifiés}

Le tableau 1 présente le nombre de systèmes à avantages concurrentiels identifiés à l'aide de la grille stratégique et ce, pour les cinq entreprises. Ce nombre varie de 10 à 19 en fonction des entreprises, pour une moyenne de 12,8 applications.

Tab. 1. Nombre de SIAC identifiés par entreprise

\begin{tabular}{|lc|}
\hline Secteur d'activité & Nombre de SIAC \\
\hline Cuir & 10 \\
Meuble & 19 \\
Papier & 10 \\
Alimentation & 15 \\
Produits chimiques & 10 \\
& \\
\hline Moyenne & 64 \\
\hline
\end{tabular}

\subsection{Types de SIAC}

La distribution de systèmes identifiés à l'intérieur de la grille stratégique présente une différence quant aux stratégies concurrentielles utilisées et aux cibles stratégiques visées (tableau 2). En effet, le nombre de systèmes varie d'une case à l'autre. La majorité d'entre eux visent une réduction des coûts face aux clients (25/ 64) ou une stratégie de différenciation vis-à-vis des concurrents (16/64). Ainsi, un plus grand nombre d'applications ont été identifiées pour répondre à une stratégie de coûts. Vient en second lieu, la stratégie de différenciation puis, finalement, la stratégie de croissance.

Tab. 2. Distribution de SIAC dans la grille stratégique $(N=64)$

\begin{tabular}{|l|r|r|r|rr|}
\hline \multirow{2}{*}{$\begin{array}{l}\text { Stratégies } \\
\text { concurrentielles }\end{array}$} & \multicolumn{3}{|c|}{ Cibles stratégiques } & \multirow{2}{*}{ Total } \\
\cline { 2 - 5 } & Fournisseurs & Concurrents & Clients & & \\
\hline Différenciation & 3 & 16 & 4 & 23 & $(35.9 \%)$ \\
Coût & 10 & 1 & 25 & 36 & $(56.3 \%)$ \\
Croissance & 0 & 5 & 0 & 5 & $(7.8 \%)$ \\
\cline { 2 - 6 } Total & 13 & 17 & 34 & 64 & $(100 \%)$ \\
\cline { 2 - 6 } & $(20.3 \%)$ & $(26.6 \%)$ & $(53.1 \%)$ & & \\
\hline
\end{tabular}




\subsection{Coûts de développement}

Tous les systèmes identifiés ont été évalués par un membre du département informatique de chaque organisation pour identifier les coûts et le temps de développement et d'implantation. Pour les cinq entreprises où fut effectuée la recherche, les coûts de développement et d'implantation des SIAC s'échelonnent de $1000 \$$ à $750000 \$$, pour un coût moyen de $69540,82 \$$ (tableau 3). Les coûts incluent entre autres l'achat d'équipements et/ou de logiciels ainsi que les coûts relatifs à la programmation. Notons que le montant $n$ 'inclut pas les dépenses d'entretien.

Tab. 3. Coats de développement et d'implantation des SIAC ( $N=64)$

\begin{tabular}{|lr|}
\hline Coût moyen & $69541 \$$ \\
Coût minimum & $1000 \$$ \\
Coût maximum & $750000 \$$ \\
\hline
\end{tabular}

\subsection{Revenus estimés}

Les participants ont été amenés, lors de l'évaluation des SIAC, à estimer de façon sommaire les revenus pouvant être retirés de l'utilisation des systèmes au sein de leur organisation. Sur la base des évaluations effectuées par les divers membres del'organisation, les revenus potentiels varient de façon considérabled'une application à l'autre, pour une moyenne de 71948 (tableau 4). Les intervenants ont cependant été dans l'impossibilité de quantifier les revenus générés pour 18 des 64 systèmes, souvent parce qu'ils ne pouvaient préciser avec suffisamment d'exactitude les retombées du système ou parce que les avantages perçus étaient de nature qualitative.

Tab. 4. Revenus estimés de l'utilisation des SIAC ( $N=48)$

\begin{tabular}{|lr|}
\hline Revenu moyen & $71948 \$$ \\
Revenu minimum & $0 \$$ \\
Revenu maximum & $560000 \$$ \\
\hline
\end{tabular}

\subsection{Décision d'implantation}

Les intentions des participants quantà l'implantation des diverses applications informatiques au sein de leur organisation sont les suivantes : les gestionnaires ont exprimé leur intérêt envers les systèmes dans $75 \%$ des cas (48 systèmes sur 64 ) alors qu'ils préfèrent ne pas les implanter dans $25 \%$ des cas, soit 16 systèmes sur 64 (tableau 5). Le nombre d'applications qui devraient être implantées dans chacune des entreprises varie de 6 à 15 , pour une moyenne de 9,6. D'autre part, le nombre moyen de SIAC qui ne semblent pas intéresser les dirigeants est de 3,2, ce nombre variant de 1 à 4 selon l'entreprise. 
Tab. 5. Implantation des SIAC ( $N=64)$

\begin{tabular}{|l|r|r|}
\hline Fréquences & $\begin{array}{r}\text { Oui } \\
(\mathrm{N}=48)\end{array}$ & $\begin{array}{r}\text { Non } \\
(\mathrm{N}=16)\end{array}$ \\
\hline Moyenne & 9,6 & 3,2 \\
Minimum & 6,0 & 1,0 \\
Maximum & 15,0 & 4,0 \\
\hline
\end{tabular}

\subsection{Période d'implantation}

La période prévue de l'implantation des systèmes est de 6 mois à 5 ans, la moyenne se situant à 2,5 ans (tableau 6). On prévoit démarrer le développement de plus de $50 \%$ des applications (27/48) à l'intérieur des deux années suivant leur identification.

Tab. 6. Moment d'Implantation des $\operatorname{SIAC}(\mathbf{N}=48)$

\begin{tabular}{|c|c|}
\hline Moment d'implantation (ans) & Nombre de SIAC (\%) \\
\hline 0,5 & 1 \\
1 & 8 \\
1,5 & 7 \\
2 & 11 \\
2,5 & 4 \\
3 & 12 \\
5 & 5 \\
\hline
\end{tabular}

\section{Conclusion}

La technologie informatique, utilisée comme arme concurrentielle, permet aux entreprises de taille moyenne de se tailler une place de choix dans leur industrie. Elle donne aux moyennes entreprises l'opportunité d'accrôtre leur compétitivité dans leur secteur d'activité. L'utilisation de la méthode de planification proposée a permis d'identifier un grand nombre d'opportunités de SIAC dans les entreprises visées sans toutefois montrer qu'elle est toujours possible ou avantageuse.. 


\section{BIBLIOGRAPHIE}

Bergeron, F, Buteau, C. et Raymond, L., (1989), «Information systems for competitive advantage : applying and comparing two methodologies», Document RIO :58, Faculté des sciences de l'administration, Université Laval.

Benjamin, R.I., Rockart, J.F., Scoth, M. et Wyman, J., (1984), «Information technology : a strategic opportunity», Sloan Management Review, vol. 25, $n^{\circ} 3$, printemps, pp. 3-10.

Clemons, E.K. et McFarlan, F.W., (1986), «Telecom: Look up or lose out», Harvard Business Review, juillet-août, pp. 91-97.

Coyne, K.P., (1986), «Sustainable competitive advantage - what it is, what it isn't», Business Horizons, janvier-février, pp. 54-61.

Dupont, C., (1986), «Les PME face aux "megatrends"», Revue Française de Gestion, n 55, janvier-février, pp. 96-105.

EDP Analyzer, (1984), «Developing strategic information systems», EDP Analyzer, vol. 22, $\mathrm{n}^{\circ}$ 5, mai, pp. 1-12.

Ives, B. et Learmonth, G.P., (1984), «The information system as a competitive weapon», Communications of the ACM, vol. 27, $\mathrm{n}^{\circ} 12$, décembre, pp. 1193-1201.

Julien, P.-A., Barrière, J. et Hébert, L., (1988), «Les facteurs de diffusion et de pénétration des nouvelles technologies dans les PME manufacturières québécoises», Revue Internationale P.M.E., vol. $1, \mathrm{n}^{\circ} 2$.

Learmonth, G.P. et Ives, B., (1987), «Information system technology can improve customer service», DataBase, hiver, pp. 6-10.

McFarlan, F.W., (1984), «Information technology changes the way you compete», Harvard Business Review, vol. 62, $\mathrm{n}^{\circ} 3$, mai-juin, pp. 98-103.

Munro, M.C. et Huff, S.L., (1985), «Information technology and corporate strategy», Business Quarterly, vol. 50, n² été, pp. 18-24.

Parsons, G.L., (1983), «Information technology : a new competitive weapon», Sloan Management Review, vol. 25, $\mathrm{n}^{\circ}$ 1, automne, pp. 2-14.

Porter, M.E., (1979), «How competitive forces shape strategy», Harvard Business Review, vol. 57, $\mathrm{n}^{\circ} 2$, mars-avril, pp. 137-145.

Porter, M.E.,(1980), Competitive strategy : techniquesfor analyzing industries and competitors, Free Press, New York.

Porter, M.E. et Millar, V.E., (1985), «How information gives you competitive advantage», Harvard Business Review, vol. 63, $\mathrm{n}^{\circ} 4$, juillet-août, pp. 149-160.

Porter, M.E., (1985a), Competitive advantage : creating and sustaining superior performance, Free Press, New York.

Porter, M.E., (1985b), «Technology and competitive advantage», The Journal of Business Strategy, vol. 5, $\mathrm{n}^{\circ} 3$, hiver, pp. 60-78.

Rackoff, N., Wiseman, C. et Ullrich, W.A., (1985), «Information systems for competitive advantage : implementation of a planning process», MIS Quarterly, vol. $9, \mathrm{n}^{\circ} 4$, décembre, pp. 285-294.

Raymond, L., (1984), «Information systems and the specificity of small business», Journal of small business-Canada, vol. $2, \mathrm{n}^{\circ}$ 2, automne, 36-2. ?

Raymond, L, Rivard, S. et Bergeron, F., (1988), L'informatisation des PME : douze cas type, L'institut de recherches politiques et les Presses de l'Université Laval, Québec.

Wiseman, C., (1985), Strategy and computers : information systems as competitive weapons, Dow Jones-Irwin Homewood, Illinois.

Wiseman, C. et MacMillan, I.C., (1984), «Creating competitive weapons from information systems», The Journal of Business Strategy, automne, pp. 42-49. 